\title{
Convergent synthesis of a blocked trisaccharide fragment related to the exopolysaccharide from Burkholderia cepacia
}

\author{
Jayant Srivastava, Anakshi Khare, and Naveen K. Khare* \\ Department of Chemistry, Lucknow University, Lucknow-226007, India \\ E-mail: nkhare58@gmail.com
}

\begin{abstract}
An effective chemical synthesis of the blocked trisaccharide fragment Gal-(1-6)-Man-(1-3)-Glu related to exopolysaccharide of Burkholderia cepacia using a convergent block synthesis approach is described. A selectively protected glucose synthon methyl 2-O-benzoyl-4,6-Obenzylidene- $O$ - $\alpha$-D-glucopyranoside (1) was condensed with ethyl 2,3,4-tri- $O$-benzyl-6- $O$ chloroacetyl-1-thio- $\alpha$-D-mannopyranoside (8) using $N$-iodosuccinimide and trimethylsilyl trifluoromethanesulfonate to give the disaccharide methyl 2,3,4-tri- $O$-benzyl-6- $O$-chloroacetyl$O$ - $\alpha$-D-mannopyranosyl-(1-3)-2- $O$-benzoyl-4,6- $O$-benzylidene- $O$ - $\alpha$-D-glucopyranoside (9). This disaccharide was selectively deprotected to give the acceptor methyl 2,3,4-tri-O-benzyl-O- $\alpha$-Dmannopyranosyl-(1-3)-2-O-benzoyl-4,6- $O$-benzylidene- $O$ - $\alpha$-D-glucopyranoside (10). Finally compound 10 was condensed with ethyl 2,3,4,6-tetra- $O$-acetyl-1-thio- $\beta$-D-galactopyranoside (11) using $\mathrm{N}$-iodosuccinimide and trimethylsilyl trifluoromethanesulfonate to give the blocked trisaccharide, methyl 2,3,4,6-tetra- $O$-acetyl- $O$ - $\beta$-D-galactopyranosyl-(1-6)-2,3,4-tri- $O$-benzyl- $O$ $\alpha$-D-mannopyranosyl-(1-3)-2-O-benzoyl-4,6- $O$-benzylidene- $O$ - $\alpha$-D-glucopyranoside (12).
\end{abstract}

Keywords: Carbohydrates, glycosylation, regioselective, stereoselective

\section{Introduction}

The important role of oligosaccharides in the biological processes ${ }^{1}$ has been recognized for a long time. Consequently, synthetic oligosaccharides have been indispensable probes for the life sciences. ${ }^{2}$ Methods for the chemical synthesis of oligosaccharides require extensive use of various regio- and stereoselective protecting strategies as these protecting groups can influence the reactivity of the electrophile and nucleophile in glycosylation reactions on the basis of steric and electronic requirements. With the objective to gain a detailed insight into the structural requirements for studying the pharmacological parameters of the biological repeating units of bacterial $O$-lipopolysaccharide, attention has been focused on the synthesis of hapten moieties 
since it has been reported that small carbohydrate haptens can afford important intermediates for structure activity studies and for building important blocks such as antigenic factors.

In this paper we report the synthesis of a blocked trisaccharide fragment of the exopolysaccharide isolated from the clinical isolate ${ }^{3}$ of Burkholderia cepacia. ${ }^{4}$ B. cepacia emerged in the early $1980 \mathrm{~s}$ as a multidrug resistant species, as a potentially important opportunistic pathogen for immunocompromised patient and a real threat for persons suffering from cystic fibrosis (CF) because of the rapid and fatal decline of pulmonary functions observed in some cases (cepacia syndrome). 5,6

\section{Results and Discussion}

The strategy envisaged the synthesis of derivatives of glucose, mannose and galactose by applying various protecting and deprotecting methodologies. For this we started with the regioselective benzoylation of methyl 4,6- $O$-benzylidene- $O$ - $\alpha$-D-glucopyranoside at the $\mathrm{C} 2$ hydroxyl group with 1-(benzyloxy) benzotriazole ${ }^{7}$ to afford methyl 2-O-benzoyl-4,6-Obenzylidene- $O-\alpha-\mathrm{D}$-glucopyranoside $\mathbf{1}$ as a crystalline product in $80 \%$ yield. This compound $\mathbf{1}$ served as an acceptor for the synthesis of the disaccharide. For the synthesis of the donor, silylation of ethyl 1-thio- $\alpha$-D-mannopyranoside ${ }^{8}$ with tert-butyldiphenylchlorosilane ${ }^{9 \mathrm{a}, \mathrm{b}}$ gave ethyl 6-O-tert-butyldiphenylsilyl-1-thio- $\alpha$-D-mannopyranoside ${ }^{10} 2$ as a syrup in $85 \%$ yield. Compound 2 on benzylation with $\mathrm{NaH}$ and $\mathrm{BnBr}{ }^{11}$ gave the unexpected product ethyl 2,3,4,6tetra- $O$-benzyl-1-thio- $\alpha$-D-mannopyranoside ${ }^{12} \mathbf{3}$ as a syrup in $80 \%$ yield, as evidenced by its ${ }^{1} \mathrm{H}$ NMR spectrum. Due to this failure, an alternative route for the synthesis of the desired donor had to be developed. Therefore, compound 2 was benzoylated with benzoyl chloride and pyridine ${ }^{13}$ to give ethyl 2,3,4-tri- $O$-benzoyl-6- $O$-tert-butyldiphenylsilyl-1-thio- $\alpha$-D-mannopyranoside 4 as a crystalline product, in $82 \%$ yield. Compound $\mathbf{4}$ was then condensed with the acceptor $\mathbf{1}$ using NIS/TMSOTf as promoter $^{14}$ to afford the disaccharide, methyl 2,3,4-tri- $O$-benzoyl-6- $O$-tertbutyldiphenylsilyl- $O$ - $\alpha$-D-mannopyranosyl-(1-3)-2-O-benzoyl-4,6-O-benzylidene- $O$ - $\alpha$-D-glucopyranoside 5 as crystals in $69 \%$ yield (Scheme 1). The ${ }^{1} \mathrm{H}$ NMR spectrum of the compound exhibited a singlet at $\delta 5.69$ for the H-1' of mannose confirming the $\alpha$-glycosidic linkage, it also showed a doublet $\left(\mathrm{J}_{1,2}=3.6 \mathrm{~Hz}\right)$, for one proton at $\delta 5.05$, for $\mathrm{H}-1$ of glucose and confirming it to be a $\alpha$-glycoside along with the other characteristic signals. It was also confirmed by its ${ }^{13} \mathrm{C}$ NMR showing the anomeric signals at 98.1 and 97.7 for the mannose and glucose respectively. This synthesized disaccharide 5 was also confirmed by its FABMS with [M] $]^{+}$at $\mathrm{m} / \mathrm{z} 1098$. In an attempt to deprotect the tert-butyldiphenysilyl group of 5 using $1 \mathrm{M}$ TBAF solution in THF ${ }^{15}$ it led to the removal of benzoyl groups probably due to the extremely basic fluoride ions ${ }^{16}$ (Scheme 1). Thus our aim to synthesize the target trisaccharide building block failed miserably. This failure has prompted us to look forward for some facile route for the synthesis of the desired trisaccharide. 

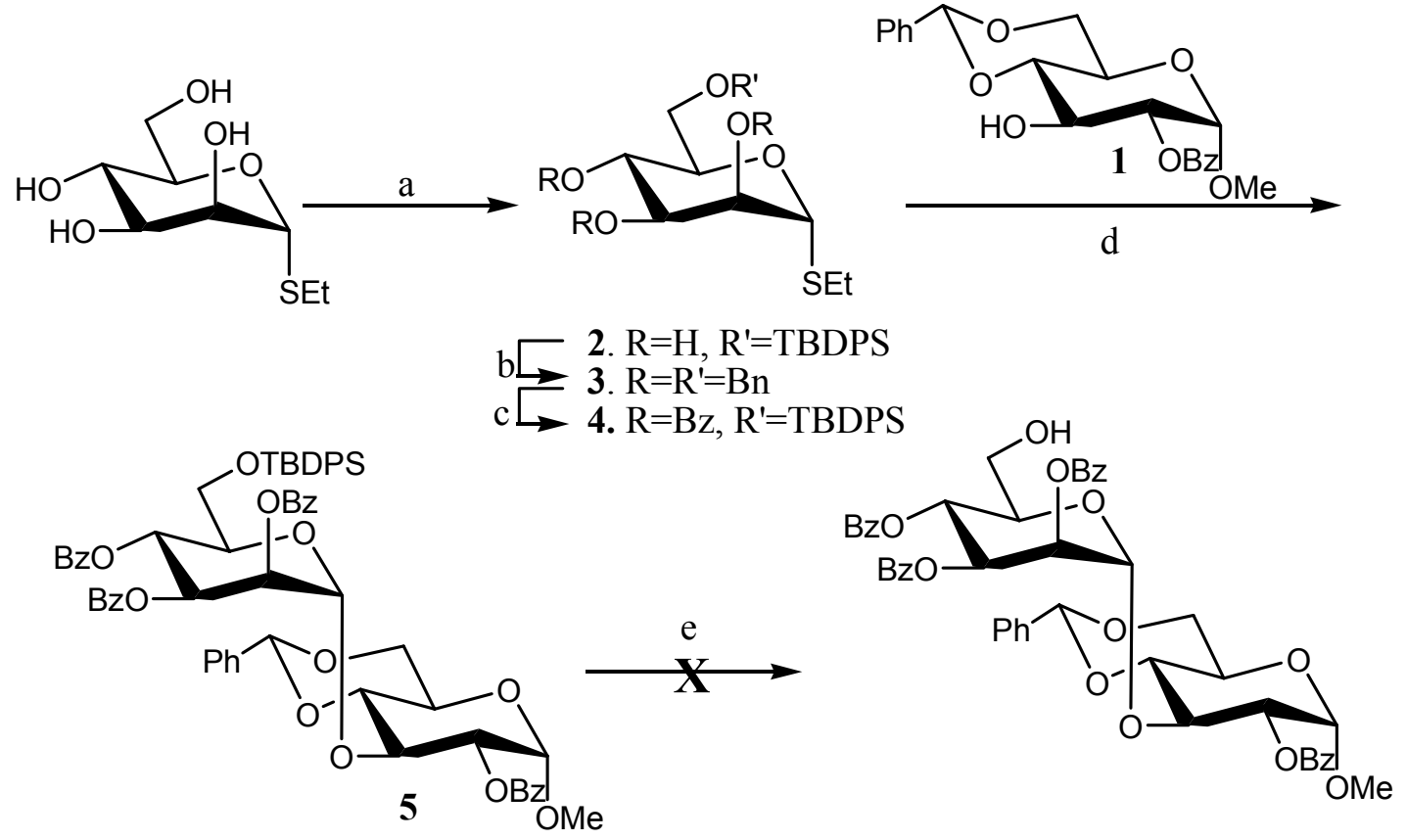

Scheme 1. (a) TBDPSC1/Pyr/DMAP/0 ${ }^{\circ} \mathrm{C} / 6$ h; 85\%; (b) $\mathrm{NaH} / \mathrm{BnBr} / \mathrm{rt} / 6 \mathrm{~h} ; 80 \%$; (c) BzCl/Pyr/ $0{ }^{\circ} \mathrm{C}, \mathrm{rt} ; 82 \%$; (d) NIS/TMSOTf $/ \mathrm{CH}_{2} \mathrm{Cl}_{2} / 4 \mathrm{~A}^{\circ} \mathrm{MS} / 0{ }^{\circ} \mathrm{C} / 20 \mathrm{~min} ; 69 \%$; (e) $1 \mathrm{M}$ TBAF in THF.

Therefore, we started with ethyl 1-thio- $\alpha$-D-mannopyranoside ${ }^{8}$ which on tritylation ${ }^{17 a, b}$ with trityl chloride and pyridine followed by the addition of $\mathrm{NaH}$ and $\mathrm{BnBr}^{11}$ gave ethyl 2,3,4-tri-Obenzyl-6- $O$-trityl-1-thio- $\alpha$-D-mannopyranoside $\mathbf{6}$ as a syrup in $80 \%$ yield. Compound 6 on detritylation with $80 \%$ aq. acetic acid $^{18}$ gave ethyl 2,3,4-tri-O-benzyl-1-thio- $\alpha$-Dmannopyranoside 7 as a syrup in $78 \%$ yield.

Compound 7 was then chloroacetylated with chloroacetyl chloride ${ }^{19}$ and pyridine to give ethyl 2,3,4-tri- $O$-benzyl-6- $O$-chloroacetyl-1-thio- $\alpha$-D-mannopyranoside $\mathbf{8}$ as a syrup in $80 \%$ yield. This compound $\mathbf{8}$ served as a donor for the synthesis of the disaccharide. Compound $\mathbf{8}$ was then condensed with acceptor 1 using NIS/TMSOTf as promoter ${ }^{14}$ to give methyl 2,3,4-tri- $O$ benzyl-6-O-chloroacetyl-O- $\alpha$-D-mannopyranosyl-(1-3)-2-O-benzoyl-4,6-O-benzylidene- $O-\alpha$-Dglucopyranoside 9 as a syrup in $69 \%$ yield (Scheme 2). Its structure was confirmed by its ${ }^{1} \mathrm{H}$ NMR spectrum showing a broad singlet for one proton at $\delta 5.36$ for the H-1' proton of mannose and a doublet for one proton at $\delta 4.98$ with $\left(\mathrm{J}_{1,2}=4.0 \mathrm{~Hz}\right)$, for $\mathrm{H}-1$ proton of glucose, and the same was also supplemented by its ${ }^{13} \mathrm{C}$ NMR peaks at 98.0 and 97.9 for the anomeric carbons of mannose and glucose respectively. This synthesized compound 9 was also confirmed by its FABMS with $[\mathrm{M}]^{+}$at $\mathrm{m} / \mathrm{z} 894$. The above disaccharide 9 was then selectively dechloroacetylated using thiourea ${ }^{19}$ to give methyl 2,3,4-tri-O-benzyl-O- $\alpha$-D-mannopyranosyl-(1-3)-2- $O$-benzoyl4,6-O-benzylidene- $O-\alpha$-D-glucopyranoside $\mathbf{1 0}$ as foam in $80 \%$ yield. Compound $\mathbf{1 0}$ served as an acceptor for the synthesis of the desired trisaccharide by condensing it with ethyl2,3,4,6-tetra- $O$ acetyl-1-thio- $\beta$-D-galactopyranoside ${ }^{8} 11$ using NIS/TMSOTf as promoter ${ }^{13}$ to give methyl 
2,3,4,6-tetra- $O$-acetyl- $O$ - $\beta$-D-galactopyranosyl-(1-6)-2,3,4-tri- $O$-benzyl- $O$ - $\alpha$-D-mannopyranosyl(1-3)-2-O-benzoyl-4,6- $O$-benzylidene- $O-\alpha$-D-glucopyranoside $\mathbf{1 2}$ as a syrup in $67 \%$ yield (Scheme 2). Its structure was confirmed by its ${ }^{1} \mathrm{H}$ NMR spectrum showing a broad singlet for one proton at $\delta 5.44$ for the $\mathrm{H}-1$ ' proton of mannose and its negligible coupling constant confirms its $\alpha$-linkage, a doublet for one proton at $\delta 5.01$ for $\mathrm{H}-1$ proton of glucose with coupling constant of $\left(\mathrm{J}_{1,2}=3.2 \mathrm{~Hz}\right)$ confirm it to be an $\alpha$-glycoside, and a doublet for one proton at $\delta 4.48$ for H-1" proton of galactose with coupling constant of $\left(\mathrm{J}_{1,2}=10.0 \mathrm{~Hz}\right)$ confirms it to be $\beta$-linked, and it was also supplemented by its ${ }^{13} \mathrm{C}$ NMR peaks at 101.4, 97.8 and 97.6 for the anomeric carbons of galactose, mannose and glucose respectively. This synthesized compound is also confirmed by its FABMS which showed $[\mathrm{M}]^{+}$at $\mathrm{m} / \mathrm{z} 1148$.
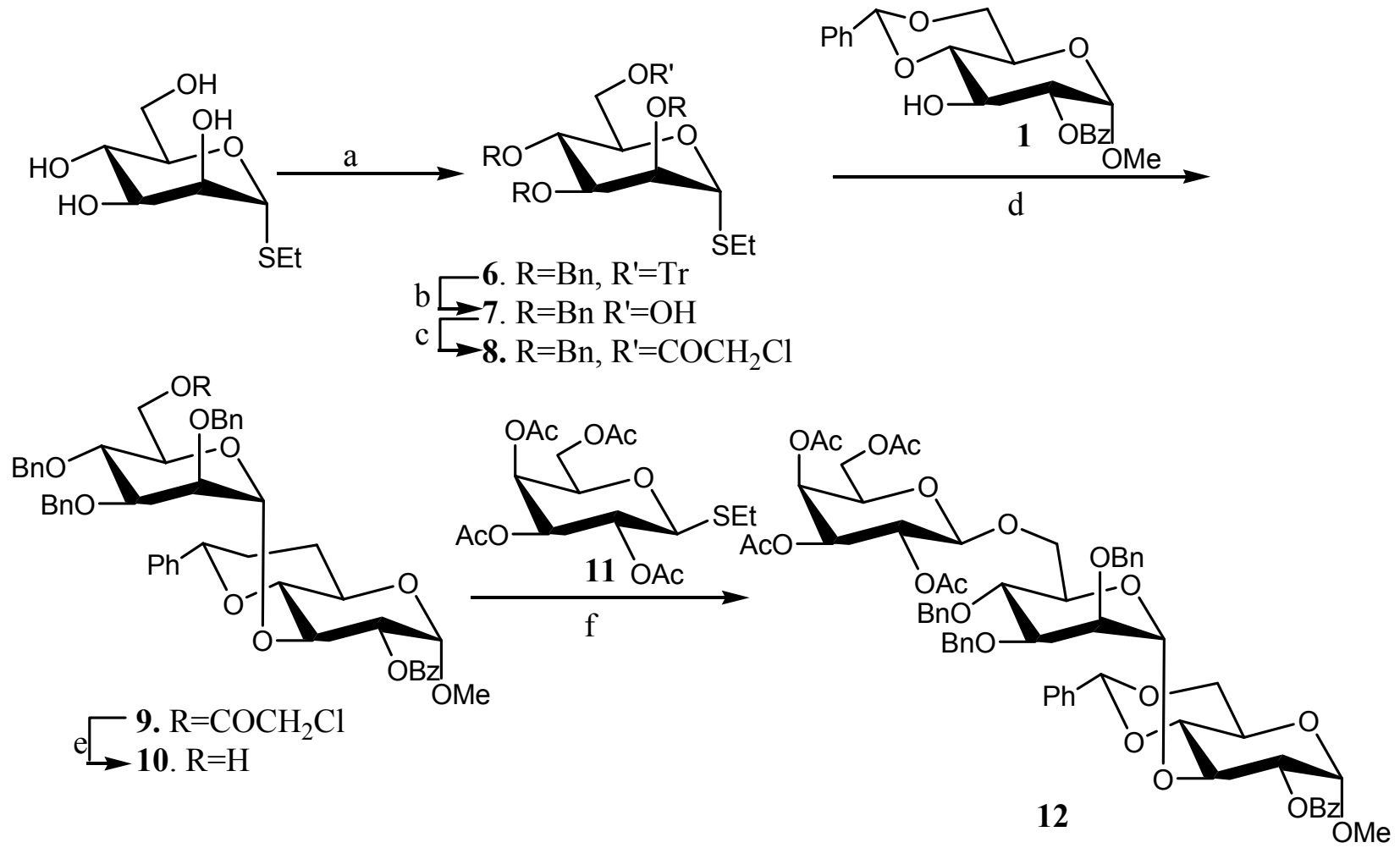

Scheme 2. (a) Trityl chloride $/ \mathrm{Pyr} / 60{ }^{\circ} \mathrm{C} / 8 \mathrm{~h} ; \mathrm{NaH} / \mathrm{BnBr} / \mathrm{DMF} / \mathrm{rt} / 4 \mathrm{~h} ; 80 \%$; (b) $80 \%$ aq. $\mathrm{AcOH}$, $80{ }^{\circ} \mathrm{C} / 6 \mathrm{~h}$; 78\%; (c) $\mathrm{ClCH}_{2} \mathrm{COCl} /$ Pyridine $0{ }^{\circ} \mathrm{C}, 6 \mathrm{~h} ; 80 \%$; (d) NIS/TMSOTf $/ \mathrm{CH}_{2} \mathrm{Cl}_{2} / \mathrm{MS}-4 \mathrm{~A} /$ $0{ }^{\circ} \mathrm{C} / 20 \mathrm{~min} ; 69 \%$; (e) Thiourea/MeOH: $\mathrm{CH}_{2} \mathrm{Cl}_{2}, 3: 2 / 3 \mathrm{~h} / \mathrm{rt} ; 80 \%$; (f) NIS/TMSOTf/ $\mathrm{CH}_{2} \mathrm{Cl}_{2} / \mathrm{MS}-$ $4 \mathrm{~A} \%{ }^{\circ} \mathrm{C} / 20 \mathrm{~min} ; 67 \%$.

The identity and stereo chemical integrity of all the intermediates and the end product described here were unambiguously confirmed by ${ }^{1} \mathrm{H},{ }^{13} \mathrm{C}$ NMR and 2D NMR experimental analysis. FAB mass spectrometry and elemental analysis provided further support for these structures. 


\section{Experimental Section}

General Procedures. All reactions were monitored by TLC on silica gel G (E. Merck). Column chromatography was performed using silica gel (SRL, 60-120 mesh). All solvents were distilled before use and evaporation was conducted at $40^{\circ} \mathrm{C}$ unless otherwise stated. Optical rotation was measured at $25^{\circ} \mathrm{C}$ on AA-5 series polarimeter. ${ }^{1} \mathrm{H}$ NMR spectra were recorded on a Bruker DPX 300 or $400 \mathrm{MHz}$ spectrometer using $\mathrm{CDCl}_{3}$ as solvent (Internal standard TMS) unless otherwise stated. Melting points were determined on Buchi 540 m.p. apparatus. Mass spectrometry were recorded on mass spectrometer model (Jeol SX 102) for FABMS.

Ethyl 2,3,4-tri- $O$-benzoyl-6-O-tert-butyldiphenylsilyl-1-thio- $\alpha$-D-mannopyranoside (4). Benzoyl chloride $(1.4 \mathrm{ml}, 11.7 \mathrm{mmol})$ was added to compound $2(1.2 \mathrm{~g}, 2.6 \mathrm{mmol})$ in dry pyridine $(6 \mathrm{ml})$ at $0^{\circ} \mathrm{C}$. The reaction mixture was stirred overnight at room temperature. Ice water (ml) was added and the reaction mixture was stirred for $1 \mathrm{~h}$. The mixture was extracted with $\mathrm{CH}_{2} \mathrm{Cl}_{2}$, washed with aq. $\mathrm{NaHCO}_{3}$ solution, with water, dehydrated over $\mathrm{Na}_{2} \mathrm{SO}_{4}$, filtered and concentrated. Chromatography (n-hexane/ethylacetate, 17:3) of the residue afforded 4 (1.6 g, $82 \%)$ as crystal, m.p. 96-98 $,[\alpha]_{\mathrm{D}}{ }^{25}-41^{\circ}\left(\mathrm{c}^{1.0} \mathrm{CHCl}_{3}\right) .{ }^{1} \mathrm{H} \mathrm{NMR}\left(\mathrm{CDCl}_{3}\right) 300 \mathrm{MHz}: \delta$ 8.15$7.16\left(\mathrm{~m}, 25 \mathrm{H}\right.$, aromatic protons), $6.16\left(\mathrm{t}, 1 \mathrm{H}, \mathrm{J}_{3,4,5}=10.2 \mathrm{~Hz}, \mathrm{H}-4\right), 5.80-5.76(\mathrm{~m}, 2 \mathrm{H}, \mathrm{H}-2, \mathrm{H}-3)$, 5.59 (bs, 1H, H-1), 4.56-4.51 (m, 1H, H-5), 3.95-3.81 (m, 2H, H-6a, 6b), 2.80-2.65 (m, 2H, $\left.\mathrm{CH}_{2} \mathrm{CH}_{3}\right), 1.35\left(\mathrm{t}, 3 \mathrm{H}, \mathrm{J}=7.5 \mathrm{~Hz},-\mathrm{CH}_{2} \mathrm{CH}_{3}\right), 1.06$ (s, 9H, tert-butyl). Anal. Calcd. for $\mathrm{C}_{45} \mathrm{H}_{46} \mathrm{O}_{8} \mathrm{SSi}: \mathrm{C}, 69.74 ; \mathrm{H}, 5.98$; found $\mathrm{C}, 69.69 ; \mathrm{H}, 5.99$.

Methyl 2,3,4-tri- $O$-benzoyl-6- $O$-tert-butyldiphenylsilyl- $O$ - $\alpha$-D-mannopyranosyl-(1-3)-2-Obenzoyl-4,6- $\boldsymbol{O}$-benzylidene- $\boldsymbol{O}$ - $\boldsymbol{\alpha}$-D-glucopyranoside (5). A mixture of 4 (400 $\mathrm{mg}, 0.52 \mathrm{mmol}$ ), and 1 (181.3 $\mathrm{mg}, 0.47 \mathrm{mmol})$ and $4 \mathrm{~A}^{0}$ molecular sieves $(600 \mathrm{mg})$ in $\mathrm{CH}_{2} \mathrm{Cl}_{2}(8 \mathrm{ml})$ was cooled under nitrogen to $0^{0}$ and stirred for $10 \mathrm{~min}$. NIS $(151 \mathrm{mg}, 0.67 \mathrm{mmol})$ and TMSOTf $(47 \mu 1,0.26$ mmol) were successively added, the mixture was stirred for $20 \mathrm{~min}$, neutralized by the addition of triethylamine and filtered through a layer of celite. The filtrate was washed with aq. $\mathrm{Na}_{2} \mathrm{~S}_{2} \mathrm{O}_{3}$ solution and with water, dehydrated over $\mathrm{Na}_{2} \mathrm{SO}_{4}$ and concentrated. Chromatography (nhexane/ethylacetate, 17:3) of the residue afforded $5(380 \mathrm{mg}, 69 \%)$ as crystal m.p. $94-96^{\circ},[\alpha]_{\mathrm{D}}{ }^{25}$

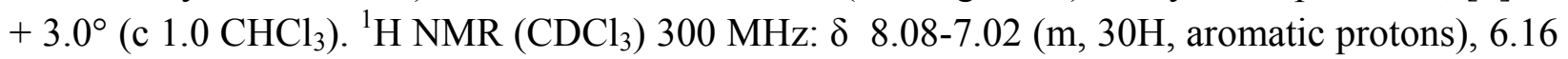
(t, 1H, J J $\left.\mathrm{CHC}_{6} \mathrm{H}_{5}\right), 5.58\left(\mathrm{~m}, 1 \mathrm{H}, \mathrm{H}-3^{\prime}\right), 5.25\left(\mathrm{dd}, 1 \mathrm{H}, \mathrm{J}_{1,2}=3.6 \mathrm{~Hz}, \mathrm{~J}_{2,3}=9.9 \mathrm{~Hz}, \mathrm{H}-2\right), 5.05$ (d, 1H, J ${ }_{1,2}=$ $3.6 \mathrm{~Hz}, \mathrm{H}-1), 4.56$ (t, 1H, J $2,3,4=9.0 \mathrm{~Hz} \mathrm{H}-3), 4.39-4.30$ (m, 2H, H-6a, 6b Glu), 4.13-4.10 (m, 1H, H-5'), 3.95-3.71 (m, 3H, H-5, H-6a, 6b Man), 3.67-3.62 (m, 1H, H-4), 3.37 (s, 3H, -OCH $)$, 1.06 (s, 9H, tert-butyl). ${ }^{13} \mathrm{C} \mathrm{NMR}\left(\mathrm{CDCl}_{3}\right): 165.7,165.6,165.1,164.7\left(4 \mathrm{X}-\mathrm{COC}_{6} \mathrm{H}_{5}\right), 136.8-$ 125.9 (Aromatic carbons), $101.1\left(-\mathrm{CHC}_{6} \mathrm{H}_{5}\right), 98.1$ (C-1', Man), 97.7 (C-1, Glu), 82.4, 72.6, 72.1, 70.9 (2C), 70.8, 70.1, 68.9, 65.8, $62.0(2 \mathrm{C}), 55.4\left(-\mathrm{OCH}_{3}\right), 26.6\left(\left[-\mathrm{C}\left(\mathrm{CH}_{3}\right)_{3}\right]\right), 19.1\left(\left[-\mathrm{C}\left(\mathrm{CH}_{3}\right)_{3}\right]\right)$. Anal. Calcd. for $\mathrm{C}_{64} \mathrm{H}_{62} \mathrm{O}_{15} \mathrm{Si}: \mathrm{C}, 69.93 ; \mathrm{H}, 5.68$; found $\mathrm{C}, 69.87 ; \mathrm{H}, 5.71$. 
Ethyl 2,3,4-tri- $\boldsymbol{O}$-benzyl-6- $\boldsymbol{O}$-trityl-1-thio-a-D-mannopyranoside (6). To a stirred solution of ethyl 1-thio- $\alpha$-D-mannopyranoside $(1.5 \mathrm{~g}, 6.7 \mathrm{mmol})$ in pyridine $(8 \mathrm{ml})$ was added trityl chloride $(2.8 \mathrm{~g}, 10.0 \mathrm{mmol})$ at $0^{\circ} \mathrm{C}$ and the reaction mixture was stirred for $8 \mathrm{~h}$ at $60^{\circ} \mathrm{C}$. The solution was dissolved in chloroform and filtered to remove excess of trityl chloride, washed with water, dried and concentrated to give ethyl-6-O-trityl-1-thio- $\alpha$-D-mannopyranoside as yellow syrup. The crude product $(2.3 \mathrm{~g}, 4.9 \mathrm{mmol})$ was dissolved in dry DMF $(15 \mathrm{ml}), \mathrm{NaH}(60 \%$ oil coated $1.5 \mathrm{~g}$, $36.7 \mathrm{mmol})$ and $\operatorname{BnBr}(2.6 \mathrm{ml}, 21.9 \mathrm{mmol})$ were added and the mixture was stirred at room temperature for $6 \mathrm{~h}$. $\mathrm{MeOH}(8 \mathrm{ml})$ was then added to destroy the excess reagents, the reaction mixture was diluted with $\mathrm{CH}_{2} \mathrm{Cl}_{2}$, washed with water, dehydrated over $\mathrm{Na}_{2} \mathrm{SO}_{4}$ and concentrated. Chromatography (n-hexane/ethylacetate, 17:3) of the residue afforded 6 as syrup (2.90 g, 80\%), $[\alpha]_{\mathrm{D}}{ }^{25}+26.8\left(\mathrm{c} 1.1, \mathrm{CHCl}_{3}\right) .{ }^{1} \mathrm{H}$ NMR $\left(\mathrm{CDCl}_{3}\right) 400 \mathrm{MHz}: \delta 8.15-7.16(\mathrm{~m}, 30 \mathrm{H}$, aromatic protons), 5.44 (bs, 1H, H-1), $4.79\left(\mathrm{~d}, 1 \mathrm{H}, \mathrm{J}=12.0 \mathrm{~Hz},-\mathrm{CH} \mathrm{HC}_{6} \mathrm{H}_{5}\right), 4.74-4.57$ (m, 4H, $2 \mathrm{X}-$ $\left.\mathrm{CH}_{2} \mathrm{C}_{6} \mathrm{H}_{5}\right), 4.27\left(\mathrm{~d}, 1 \mathrm{H}, \mathrm{J}=10.4 \mathrm{~Hz},-\mathrm{CH} H C_{6} \mathrm{H}_{5}\right), 4.14\left(\mathrm{dd}, 1 \mathrm{H}, \mathrm{J}_{2,3}=3.2 \mathrm{~Hz}, \mathrm{~J}_{3,4}=10.0 \mathrm{~Hz}, \mathrm{H}-\right.$ 3), 4.07 (t, 1H, J $\left.\mathrm{J}_{3,4,5}=9.2 \mathrm{~Hz}, \mathrm{H}-4\right), 3.84-3.82$ (m, 2H, H-5, H-2), 3.49, (d, 1H, J 6 a,6b $=9.6 \mathrm{~Hz}, \mathrm{H}-$ 6a), $3.29\left(\mathrm{dd}, 1 \mathrm{H}, \mathrm{J}_{5,6 \mathrm{~b}}=4.8 \mathrm{~Hz}, \mathrm{~J}_{6 \mathrm{a}, 6 \mathrm{~b}}=9.6 \mathrm{~Hz}, \mathrm{H}-6 \mathrm{~b}\right), 2.72-2.56\left(\mathrm{~m}, 2 \mathrm{H},-\mathrm{CH}_{2} \mathrm{CH}_{3}\right), 1.28(\mathrm{t}, 3 \mathrm{H}$, $\mathrm{J}=7.6 \mathrm{~Hz},-\mathrm{CH}_{2} \mathrm{CH}_{3}$ ). Anal. Calcd. for $\mathrm{C}_{48} \mathrm{H}_{48} \mathrm{O}_{5} \mathrm{~S}$ : C, 78.23; H, 6.56; found $\mathrm{C}$, 78.16; 6.61.

Ethyl 2,3,4-tri-O-benzyl -1-thio-o-D-mannopyranoside (7). A solution of 6 (2.0g, $2.7 \mathrm{mmol})$ in acetic acid : water $(4: 1,20 \mathrm{ml})$ was warmed at $80^{\circ} \mathrm{C}$ for $1 \mathrm{~h}$. The reaction mixture was then concentrated by co-evaporation of toluene under reduced pressure. Chromatography (nhexane/ethylacetate, $8: 2)$ of the residue afforded $7(1.1 \mathrm{~g} 78 \%)$ as syrup, $[\alpha]_{\mathrm{D}}{ }^{25}+61^{\circ}(\mathrm{c} 1.8$, $\left.\mathrm{CHCl}_{3}\right) .{ }^{1} \mathrm{H}$ NMR $\left(\mathrm{CDCl}_{3}\right) 400 \mathrm{MHz}: \delta$ 7.37-7.24 (m, 15H, aromatic protons), 5.29 (bs, $1 \mathrm{H}, \mathrm{H}-$ 1), $4.93\left(\mathrm{~d}, 1 \mathrm{H}, \mathrm{J}=11.2, \mathrm{~Hz},-\mathrm{CHHC}_{6} \mathrm{H}_{5}\right), 4.76-4.58\left(\mathrm{~m}, 5 \mathrm{H}, 5 \mathrm{X}-\mathrm{CH} H \mathrm{C}_{6} \mathrm{H}_{5}\right), 4.03-3.98(\mathrm{~m}, 2 \mathrm{H}$, H-2, H-3), 3.87-3.70 (m, 4H, H-4, H-5, H-6a, 6b), 2.64-2.47 (m, 2H, - $\left.\mathrm{CH}_{2} \mathrm{CH}_{3}\right), 1.22$ (t, 3H, J = 7.6 Hz, $-\mathrm{CH}_{2} \mathrm{CH}_{3}$ ). Anal. Calcd. for $\mathrm{C}_{29} \mathrm{H}_{34} \mathrm{O}_{5} \mathrm{~S}: \mathrm{C}, 70.42 ; \mathrm{H}, 6.93$; found $\mathrm{C}$, 70.35; H, 6.97 .

Ethyl 2,3,4-tri- $\boldsymbol{O}$-benzyl-6- $\boldsymbol{O}$-chloroacetyl-1-thio- $\boldsymbol{\alpha}$-D-mannopyranoside (8). Chloro- acetyl chloride $(0.26 \mathrm{ml}, 3.2 \mathrm{mmol})$ was added dropwise at $0^{\circ}$ to the solution of compound $7(1.0 \mathrm{~g}, 2.0$ $\mathrm{mmol})$ and pyridine $(0.2 \mathrm{ml}, 2.6 \mathrm{mmol})$ in $\mathrm{CH}_{2} \mathrm{Cl}_{2}(20 \mathrm{ml})$. The mixture was stirred for $16 \mathrm{~h}$ at room temperature and poured into water. The organic layer was separated, washed with aq. $\mathrm{HCl}$ $(10 \%, \mathrm{v} / \mathrm{v})$ and aq. $\mathrm{NaHCO}_{3}$ solution $(8 \%$, w/v), dried and concentrated. Chromatography (nhexane/ethylacetate $9: 1)$ of the residue afforded 8 as syrup $(935 \mathrm{mg}, 80 \%),[\alpha]_{\mathrm{D}}{ }^{25}+64^{\circ}(\mathrm{c} 1.4$ $\left.\mathrm{CHCl}_{3}\right) .{ }^{1} \mathrm{H} \mathrm{NMR}\left(\mathrm{CDCl}_{3}\right) 400 \mathrm{MHz}: \delta$ 7.37-7.24 (m, 15H, aromatic protons), 5.33 (bs, 1H, H1), $4.93\left(\mathrm{~d}, 1 \mathrm{H}, \mathrm{J}=10.8 \mathrm{~Hz},-\mathrm{CH}_{2} \mathrm{C}_{6} \mathrm{H}_{5}\right), 4.68-4.55\left(\mathrm{~m}, 5 \mathrm{H}, 5 \mathrm{X}-\mathrm{CH} \mathrm{HC}_{6} \mathrm{H}_{5}\right), 4.41-4.37$ (m, 2H, H-2, H-3), 4.20-4.16 (m, 1H, H-5), $3.99 \& 3.98$ (2s, $1 \mathrm{H}$ each, $-\mathrm{COCH}_{2} \mathrm{Cl}$ ), 3.93 (t, 1H, J3,4 $=9.6$ $\mathrm{Hz}, \mathrm{H}-4), 3.85-3.78$ (m, 2H, H-6a, 6b), 2.70-2.49 (m, 2H, $-\mathrm{CH}_{2} \mathrm{CH}_{3}$ ), 1.24 (t, 3H, J = 7.6 Hz, $\mathrm{CH}_{2} \mathrm{CH}_{3}$ ). Anal. Calcd. for $\mathrm{C}_{31} \mathrm{H}_{35} \mathrm{Cl} \mathrm{O}_{6} \mathrm{~S}: \mathrm{C}, 65.19 ; \mathrm{H}, 6.18$; found $\mathrm{C}, 65.13 ; \mathrm{H}, 6.22$.

Methyl 2,3,4-tri- $O$-benzyl-6- $O$-chloroacetyl- $O$-a-D-mannopyranosyl-(1-3)-2- $O$-benzoyl- 4,6$\boldsymbol{O}$-benzylidene- $\boldsymbol{O}$ - $\boldsymbol{\alpha}$-D-glucopyranoside (9). A mixture of 8 (400 mg, $0.7 \mathrm{mmol})$, and 1 (246 
$\mathrm{mg}, 0.64 \mathrm{mmol})$ and $4 \mathrm{~A}^{0}$ molecular sieves $(600 \mathrm{mg})$ in $\mathrm{CH}_{2} \mathrm{Cl}_{2}(8 \mathrm{ml})$ was cooled under nitrogen to $0^{0}$ and stirred for $10 \mathrm{~min}$. NIS $(205 \mathrm{mg}, 0.91 \mathrm{mmol})$ and TMSOTf $(64 \mu 1,0.35 \mathrm{mmol})$ were successively added, the mixture was stirred for $20 \mathrm{~min}$, neutralized by the addition of triethylamine and filtered through a layer of celite. The filterate was washed with aq. $\mathrm{Na}_{2} \mathrm{~S}_{2} \mathrm{O}_{3}$ solution and with water, dehydrated over $\mathrm{Na}_{2} \mathrm{SO}_{4}$ and concentrated. Chromatography (nhexane:ethylacetate, 17:3) of the residue afforded $9(466 \mathrm{mg}, 69 \%)$ as syrup, $[\alpha]_{\mathrm{D}}{ }^{25}=+58.5^{\circ}$ (c 0.9, $\left.\mathrm{CHCl}_{3}\right) .{ }^{1} \mathrm{H} \mathrm{NMR}\left(\mathrm{CDCl}_{3}\right) 400 \mathrm{MHz}: \delta$ 8.04-6.99 (m, 25H, aromatic protons), $5.54(\mathrm{~s}, 1 \mathrm{H},-$ $\left.\mathrm{CHC}_{6} \mathrm{H}_{5}\right), 5.36$ (bs, $\left.1 \mathrm{H}, \mathrm{H}-1^{\prime}\right), 5.12\left(\mathrm{dd}, 1 \mathrm{H}, \mathrm{J}_{1,2}=3.6 \mathrm{~Hz}, \mathrm{~J}_{2,3}=10.0 \mathrm{~Hz}, \mathrm{H}-2\right), 4.98\left(\mathrm{~d}, 1 \mathrm{H}, \mathrm{J}_{1,2}=\right.$ $4.0 \mathrm{~Hz}, \mathrm{H}-1), 4.72\left(\mathrm{~d}, 1 \mathrm{H}, \mathrm{J}=11.2 \mathrm{~Hz},-\mathrm{CH} H \mathrm{C}_{6} \mathrm{H}_{5}\right), 4.54-4.42\left(\mathrm{~m}, 5 \mathrm{H}, 5 \mathrm{X}-\mathrm{CH} \mathrm{C}_{6} \mathrm{H}_{5}\right), 4.34-$ 4.29 (m, 2H, H-2', H-3'), 4.22-4.18 (m, 1H, H-5'), 3.98 \& 3.93 (2s, $1 \mathrm{H}$ each, $\left.-\mathrm{COCH}{ }_{2} \mathrm{Cl}\right), 4.00-$ 3.89 (m, 2H, H-6a, 6b Glu), 3.84-3.60 (m, 5H, H-3, H-4, H-4', H6'a, 6b' Man), 3.41 (s, 3H, $\left.\mathrm{OCH}_{3}\right) \cdot{ }^{13} \mathrm{C} \mathrm{NMR}\left(\mathrm{CDCl}_{3}\right): 167.1\left(-\mathrm{COCH}_{2} \mathrm{Cl}\right), 165.8\left(-\mathrm{COC}_{6} \mathrm{H}_{5}\right), 138.3-126.1$ (Aromatic carbons), $101.9\left(-\mathrm{CHC}_{6} \mathrm{H}_{5}\right), 98.0$ (C-1', Man), 97.9 (C-1, Glu), 82.5, 79.6, 74.6, 74.4, 73.4, 72.6, $72.0,71.9(2 \mathrm{C}), \quad 71.7,69.9,64.5,62.1,55.5\left(-\mathrm{OCH}_{3}\right), 40.7\left(-\mathrm{COCH}_{2} \mathrm{Cl}\right)$. Anal. Calcd. for $\mathrm{C}_{50} \mathrm{H}_{51} \mathrm{ClO}_{13}$ : C, 67.07; $\mathrm{H}, 5.74$; found $\mathrm{C}, 67.01 ; \mathrm{H}, 5.79$.

Methyl 2,3,4-tri- $O$-benzyl- $O$ - $\alpha$-D-mannopyranosyl-(1-3)-2- $O$-benzoyl-4,6- $O$-benzylidene- $O$ $\boldsymbol{\alpha}$-D-glucopyranoside (10). A solution of $9(400 \mathrm{mg}, 0.48 \mathrm{mmol})$ and thiourea (170 $\mathrm{mg}, 2.2$ $\mathrm{mmol})$ in a mixture of $\mathrm{MeOH}(9 \mathrm{ml})$ and $\mathrm{CH}_{2} \mathrm{Cl}_{2}(6 \mathrm{ml})$ was stirred at room temperature for $3 \mathrm{~h}$ and concentrated. The residue was dissolved in $\mathrm{CH}_{2} \mathrm{Cl}_{2}$, washed with water, dried and concentrated. Chromatography (n-hexane/ethylacetate 8:2) of the residue afforded $\mathbf{1 0}(293 \mathrm{mg}$, $80 \%)$ as foam, $[\alpha]_{\mathrm{D}}{ }^{25}+47^{\circ}\left(\mathrm{c} 1.0, \mathrm{CHCl}_{3}\right) .{ }^{1} \mathrm{H} \mathrm{NMR}\left(\mathrm{CDCl}_{3}\right) 400 \mathrm{MHz}: \delta 8.07-7.03(\mathrm{~m}, 25 \mathrm{H}$, aromatic protons), $5.56\left(\mathrm{~s}, 1 \mathrm{H},-\mathrm{CHC}_{6} \mathrm{H}_{5}\right), 5.36\left(\mathrm{bs}, 1 \mathrm{H}, \mathrm{H}-1^{\prime}\right), 5.09\left(\mathrm{dd}, 1 \mathrm{H}, \mathrm{J}_{1,2}=4.0 \mathrm{~Hz}, \mathrm{~J}_{2,3}=\right.$ $10.0 \mathrm{~Hz}, \mathrm{H}-2), 5.01$ (d, 1H, J $\left.\mathrm{J}_{1,2}=4.0 \mathrm{~Hz}, \mathrm{H}-1\right), 4.74$ (d, 1H, J = 11.2 Hz, $\left.-\mathrm{CH} H C_{6} \mathrm{H}_{5}\right), 4.53-4.41$ (m, 5H, 5 X-CHHC${ }_{6} \mathrm{H}_{5}$ ), 4.38-4.31 (m, 2H, H-2', H-3'), 3.97-3.48 (m, 9H, H 5', H-5, H-6a, 6b Glu, H-3, H-4, H-4', H-6a', 6b' Man), 3.40 (s, 3H, $\left.-\mathrm{OCH}_{3}\right) .{ }^{13} \mathrm{C} \mathrm{NMR}\left(\mathrm{CDCl}_{3}\right)$ : $165.9\left(-\mathrm{COC}_{6} \mathrm{H}_{5}\right)$, 138.5-126.1 (Aromatic carbons), 101.8 (- $\left.\mathrm{CHC}_{6} \mathrm{H}_{5}\right), 98.0$ (C-1', Man), 97.9 (C-1, Glu), 82.5, 79.4, 75.0, 74.5, 74.2, 72.6, 72.1 (3C), 71.8, 68.8, 61.9 (2C), $55.5\left(-\mathrm{OCH}_{3}\right)$. Anal. Calcd.for $\mathrm{C}_{48} \mathrm{H}_{50} \mathrm{O}_{12}$ : C, 70.40; H, 6.15; found C, 70.33; H, 6.19.

\section{Methyl 2,3,4,6-tetra- $O$-acetyl- $O$ - $\beta$-D-galactopyranosyl-(1-6)-2,3,4-tri- $O$-benzyl- $O$ - $\alpha$-D- mannopyraranosyl-(1-3)-2- $O$-benzoyl-4,6- $O$-benzylidene- $O$ - $\alpha$-D-glucopyranoside (12). A} mixture of ethyl 2,3,4,6-tetra- $O$-acetyl-1-thio- $\beta$-D-galactopyranoside (53 mg, $0.13 \mathrm{mmol}$ ), 11 (100 mg, $0.12 \mathrm{mmol}$ ) and $4 \mathrm{~A}^{0}$ molecular sieves $(150 \mathrm{mg})$ in $\mathrm{CH}_{2} \mathrm{Cl}_{2}(4 \mathrm{ml})$ was cooled under nitrogen to $0^{0}$ and stirred for $10 \mathrm{~min}$. NIS ( $40 \mathrm{mg}, 0.17 \mathrm{mmol}$ ) and TMSOTf (12 $\mu \mathrm{l}, 0.07 \mathrm{mmol}$ ) were successively added, the mixture was stirred for $20 \mathrm{~min}$, neutralized by the addition of triethylamine and filtered through a layer of celite. The filtrate was washed with aq. $\mathrm{Na}_{2} \mathrm{~S}_{2} \mathrm{O}_{3}$ solution and with water, dehydrated over $\mathrm{Na}_{2} \mathrm{SO}_{4}$ and concentrated. Chromatography (hexane:ethylacetate, 17:3) of the residue afforded $12(97 \mathrm{mg}, 67 \%)$ as syrup, $[\alpha]_{\mathrm{D}}{ }^{25}+35^{\circ}$ (c 1.0, $\left.\mathrm{CHCl}_{3}\right) . \quad{ }^{1} \mathrm{H} \mathrm{NMR}\left(\mathrm{CDCl}_{3}\right) 400 \mathrm{MHz}: \delta 8.09-6.97(\mathrm{~m}, 25 \mathrm{H}$, aromatic protons), $5.56(\mathrm{~s}, 1 \mathrm{H}$, 
$-\mathrm{CHC}_{6} \mathrm{H}_{5}$ ), 5.44 (bs, 1H, H-1'), 5.32 (d, 1-H, J =2.8 Hz, H-4"), 5.27 (t, 1H, J,2,3 = 9.6 Hz H-2"), 5.08 (dd, $\left.1 \mathrm{H}, \mathrm{J}_{2,3}=10.0 \mathrm{~Hz}, \mathrm{~J}_{3,4}=3.2 \mathrm{~Hz} \mathrm{H}-3 "\right), 5.01$ (d, 1H, J $\left.\mathrm{J}_{1,2}=3.2 \mathrm{~Hz}, \mathrm{~J}_{2,3}=10.8 \mathrm{~Hz}, \mathrm{H}-2\right), 4.75\left(\mathrm{~d}, 1 \mathrm{H}, \mathrm{J}=11.6 \mathrm{~Hz},-\mathrm{CH} H \mathrm{C}_{6} \mathrm{H}_{5}\right), 4.48\left(\mathrm{~d}, 1 \mathrm{H}, \mathrm{J}_{1,2}=10.0\right.$ Hz, H-1"), 4.44-4.36 (m, 4H, $2 \mathrm{X}-\mathrm{CH}_{2} \mathrm{C}_{6} \mathrm{H}_{5}$ ), 4.35-4.30 (m, 2H, H-2', H-3'), 4.26-4.24 (m, 1H, $\mathrm{H}-5), 4.23$ (d, 1H, J = 12.0 Hz, $-\mathrm{CHHC}_{6} \mathrm{H}_{5}$ ), 4.13-4.01 (m, 2H, H-6a", 6b" Gal), 3.98-3.96 (m, 2H, H-6a, 6b Glc), 3.82-3.67 (m, 7H, H-3, H-4, H-4', H-5', H-5", H6a', 6b' Man), 3.41 (s, 3H, $\left.\mathrm{OCH}_{3}\right), 2.11,2.02,1.95,1.93\left(\mathrm{~s}, 3 \mathrm{H}\right.$ each, $\left.4 \mathrm{X}-\mathrm{OCOCH}_{3}\right) .{ }^{13} \mathrm{C} \mathrm{NMR}\left(\mathrm{CDCl}_{3}\right): 170.3,170.2$, 169.1, 165.9 (4 X- $\left.\mathrm{COCH}_{3}\right), 138.8-126.11$ (Aromatic carbons), 101.9(-CHC $\left.6 \mathrm{H}_{5}\right), 101.4(\mathrm{C}-1$ ", Gal), 97.8 (C-1', Man), 97.6 (C-1, Glu), 82.7, 79.5, 74.4, 74.1 (2C), 72.1, 71.9, 71.7(2C), 71.1 (2C), 70.4, 69.0, 68.7, 68.3, 67.1, 61.9, 61.2, $\left.55.4\left(-\mathrm{OCH}_{3}\right), 29.6\left(-\mathrm{COCH}_{3}\right), 20.63 \mathrm{X}-\mathrm{COCH}_{3}\right)$. Anal. Calcd. for $\mathrm{C}_{62} \mathrm{H}_{68} \mathrm{O}_{21}: \mathrm{C}, 64.80 ; \mathrm{H}, 5.96$; found $\mathrm{C}, 64.73 ; \mathrm{H}, 6.01$.

\section{Acknowledgements}

One of us (J. S.) thanks C.S.I.R. (New Delhi, India) for financial assistance in the form of Junior and Senior Research Fellowship.

\section{References}

1. Varki, A. Glycobiology 1993, 3, 97.

2. Synthetic Oligosaccharides. Indispensable Probes in the Life Sciences: Kovac, P. Ed.; American Chemical Society: Washington, DC, 1994; Vol. 560.

3. Cerantola S.; Lemassu-Jacquier, A. and Mantrozier H. Eur. J. Biochem. 1999, $260,373$.

4. Burkholder. Phytopathology 1950, 40, 115.

5. Isles, A.; MacLusky, I.; Corey, M.; Gold, R.; Prober, C.; Fleming, P.; Levison, H. J. Pediatr. 1984, 104, 206.

6. Tablan, O. C.; Martone, W. J.; Doershuk, C. F.; Stern, R. C.; Thomassen, M. J.; Klinger, J. D.; White, J. W.; Carson, L. A. and Jarvis, W. R. Chest 1987, 91, 527.

7. Sunggak K.; Heung Chang; Wan Joo K. J. Org. Chem. 1985, 50, 1751.

8. Contour, M.O.; Defaye, J.; Little, M.; Wong, E. Carbohyr.Res. 1989, 193, 283.

9. (a) Ireland, R. E. and Obrecht, D. M. Helv. Chim. Acta., 1986, 69, 1273. (b) Clode, D. M.; Laurie, W. A.; McHale, D.; Sheriden, J. B. Carbohydr. Res. 1985, 139, 161.

10. McGavin, R. S. Org. \& Biomol. Chem. 2005, 3(15), 2723.

11. Ferrier, R. J.; Hannaford, A. J.; Overend, W. G.; Smith, B. C. Carbohydr. Res. 1965, 1, 38.

12. Saksena, R; Zhang, J.; Kovac, P. J. Carbohydr. Chem. 2002, 21(6), 453.

13. Fletcher, Jr., H. G. Meth. Carbohydr. Chem. 1963, 2, 228.

14. Demchenko, A.; Stauch, T.; Boons, G. J. Synlett. 1997, 818.

15. Hanessian, S.; Lavallee P. Can. J. Chem.1975, 53, 2975. 
16. Clark, J. H. Chem. Rev. 1980, 80, 429.

17. (a) Kovac, P.; Glaudeman, C. P. J. J. Carbohydr. Chem. 1983, 2, 313. (b) Nair, V and Chun Byoung-Kwon. ARKIVOC 2003, (i), 9.

18. Blickenstaff, R. T. J. Am. Chem. Soc. 1960, 82, 3673.

19. Lemanski, G.; Ziegler, T. Eur. J. Org. Chem. 2000, 181. 